\title{
Online Attendance Marking System Using Facial Recognition and Intranet Connectivity
}

\author{
Vibhanshu Pant ${ }^{\mathrm{a}, 1}$, Deepak Sharma ${ }^{\mathrm{a}}$, Annapurani. K ${ }^{\mathrm{a}}$, R. Sundar ${ }^{\mathrm{b}}$ \\ ${ }^{a}$ Department of Computer Science and Engineering, SRM Institute of Science and Technology, \\ Kattankulathur, India \\ ${ }^{\mathrm{b}}$ Scientist-D, National Institute of Ocean Technology, Chennai, India
}

\begin{abstract}
Keeping up the attendance record with everyday exercises is a difficult task. The conventional method of marking staff attendance is by tapping their ID card and then using fingerprint scanner. But due to COVID-19 pandemic the attendance system of using fingerprint scanner is stalled and currently not in use. The following system depends on face recognition and intranet connectivity to keep up attendance record of facilities and staff. The paper discusses the attendance marking system that is passive (no direct contact with the scanner or sensor) and restricting the users within certain network. The main goal of this system is divided in two steps, in initial step face is snare from the front camera of the smart phone and it is then recognized in the picture and in the second step these distinguished appearances and features are contrasted with stored information in data set for confirmation.
\end{abstract}

Keywords. Attendance, appearances, COVID-19, face-recognition, intranet connectivity, front-camera, data-set, database

\section{Introduction}

Maintaining attendance is a very important task in all the institutes and organizations, whether it is of students or for faculties, staff or employees. Every organization has their own method for marking attendance. Some of them are taking attendance using the old paper or file based method while others are using bio metric techniques. These methods take time for marking attendance and this could lead to large queue every day. Bio metric systems, used for attendance marking system, consists of two major process, first special highlights of an individual are stored in data set and secondly attendance is marked based on ID and confirmation of the individual. When an individual gives his attendance to the machine, it is contrasted with the data that is in the data set and in the event that it is coordinated, the attendance is marked effectively. Numerous strategies have been proposed for facial identification and acknowledgment like Ada Boost calculation, the Float Boost calculation, the S-Ada Boost calculation Support Vector Machines (SVM), and the Bayes classifier.

\footnotetext{
${ }^{1}$ Vibhanshu Pant, Department of Computer Science and Engineering, SRM Institute of Science and Technology, Kattankulathur, Email:vs3137@srmist.edu.in.
} 
Face recognition procedures can be either Appearance Based, in which utilization of surface highlights is applied to entire face or some particular districts is executed, or Feature Based, in which utilization of mathematical highlights like mouth, nose, eyes, eye temples, cheeks and connection between them is actualized.

The attendance system proposed in this paper depends upon two steps, in initial step face is snare from the front camera of the smart phone and it is then recognized in the picture and in the second step these distinguished appearances and features are contrasted with stored information in data set for confirmation. A successful message is displayed if the verification process is successful.

\section{Literature Survey}

Everybody needs to keep track of the employees that are working under them. In this area we have seen many changes, starting from using pen and paper to many RFID machines for marking daily attendance. Some organizations uses RFID cards for swiping to mark their attendance, it has its own shortcomings, like the staff have to stand in queues for a long time and there can be chances of proxy attendance also. Many organizations use fingerprint scanners in addition to RFID cards to eliminate these shortcomings.

In [4] the authors have proposed a system which uses HAAR Classifier and computer vision algorithm to implement face recognition System. The result was that when the system is integrated with the already existing system it uses the existing feature from Learning Management System, but the shortcomings of this paper were that the recognition rate is $56 \%$ In paper [5] the authors use PCA and ANN for facial recognition. They first apply the PCA algorithm for training data and reduce its dimensionality and then use ANN to classify these input data which then helps to find the pattern. The result they concluded is that the accuracy is high due to the combination of PCA with ANN as ANN used for classification is more accurate than PCA with Eigen face. The only limitation of this system is that it requires a high computational time and thus the implementation cost increases. In paper [6] the authors use PCA along with MATLAB to implement a face recognition system. The result from this system was that the pre-processing of the image and cropping the region of interest from it made the accuracy of the system higher. The drawback of this system was that this system was only tested with a single image. In paper [3] the authors use Eigenvector and Eigenvalue for designing a face recognition system. Besides using these, the system was also able to prevent the fake attendance mishap by the implementation of clock time. It is used for checking whether the student was there in the class for the whole time or not. The proposed system has many upsides but the accuracy of recognition of faces is high only for the frontal face of the student. In case the face is in the direction of 54 degree the accuracy is only 58\%. In paper [7] the authors uses PCA with Histogram due to which additional noises were removed from the data. The proposed system provides better accuracy due to the implementation of the Ada-Boost algorithm on Face Detection. In addition to this skin classification was also utilized for improving the precision of the system. The database is also updated up to date with new images. Since it does not implement the concept of clock time therefore there will be an issue of fake attendance if the student does not attend the whole class and leaves in the middle. 


\section{Proposed Face Recognition based Attendance Marking System}

We are building a mobile application to provide a hassle-free and proxy free attendance marking during the rush hour and also try to provide an alternative to fingerprint scanner for daily attendance of staffs or employees of an organisation.

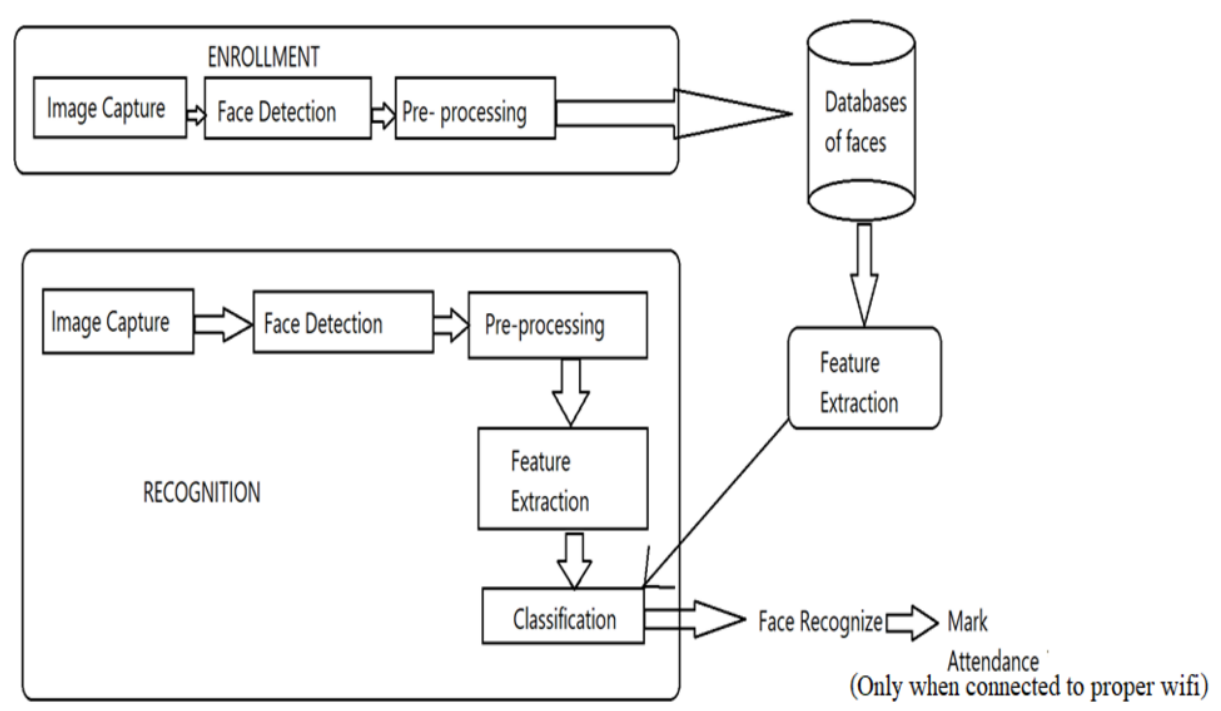

Figure 1. Architectural diagram of the system

Figure 1 shows the architectural diagram of the system demonstrating how the attendance system will work.

\subsection{Conceptual Working}

The working of the system is of two steps. The first step includes the database creation. In this, the staffs of that particular organization have to get enrolled in the system. Here an admin enrols the staff into the database, when the database is ready then only the training of the model is done over these data sets. The model is then tested with real time images.

Now the staffs have been successfully registered in the system. In the next step, they have to open the mobile application and login with the given credentials. If it is validated, the system goes to the photo capturing stage where staff clicks his photo using the front camera and the face is detected and recognized from it. This is done with the pre-existing data sets in the database. If the recognition stage is successfully passed, a message stating that the attendance has been marked is displayed on the screen.

\subsubsection{Dataset}

For training our model we have taken fifty people selfies from thirty different 
angles so totally 1500 images are taken. Live data set is being used to train our model. A copy of each selfie is then converted to grey scale. Small data set [2] used for analysis of skin tumour.

\subsubsection{Face Detection and Recognition}

For detecting and recognizing the face in the image captured by the camera Haar Cascade [1] is used. Haar Feature Selection it divides the group of pixels into two groups consisting of a black and white group. It has three variants which are edge features, line features, and four rectangular features. In this, these rectangle features are superimposed on the image array that is obtained and then calculate the data value and then move to the next one. By doing this 'delta' value is calculated for each feature which is the difference be-tween the white region from black region. In the HAAR features, the dark pixels or region describes lower values whereas the bright pixels denote higher values. In Adaboost training misclassification value is calculated by adding all the correctly classified parts and the incorrectly classified parts, these are shown by the plus sign and negative sign respectively. In Cascade classifier, all the three stages are combined and then the resultant is trained. There are four feature and each feature have different sizes and positions therefore in total HAAR Cascade Classifier has around $180000+$ features

\subsubsection{Intranet Connectivity}

To restrict the application to a certain network, WifiManager API is used which a library is provided by Android Studio. Through this, IP address of the currently connected WiFi is obtained when IP Address function from connection Info class of System Service package is invoked. It is checked against the IP Address of the network we want the application to work in. If it doesn't match, an error message is shown otherwise login activity is continued.

\subsubsection{Database}

Firebase Firestore is used as a database. We save the attendance in name and timestamp format. The attendance can be later download as json file. We selected this as our database since it is scalable and follows ACID properties and it is also integrated with google cloud for better storage.

\section{Results and Discussion}

To build the android application Android Studio is used and for building face detection and recognition model Pycharm is used as Integrated Development Environment (IDE). JAVA, Python and Kotlin are used as programming languages.

\subsection{Face Detection}

Face detection is the first step for face recognition and it can be done by four different methods as Knowledge based, Feature based, Template matching and Appearance based. In our work we have gone with Feature based technique. 
The Feature Based technique utilizes a pre-processor of the picture based technique and aides the hunt of picture based strategies utilizing GUI that inspect the face competitor locales rather than performing colossal inquiry in all aspects of the test picture. In this we are using Haar cascade classifier for face detection and it is Feature based, and with edge detection, centre detection, line detection, centre detection and facial features like eyes nose mouth. Figure 2 shows the outcome when python scripts are run for detecting faces.

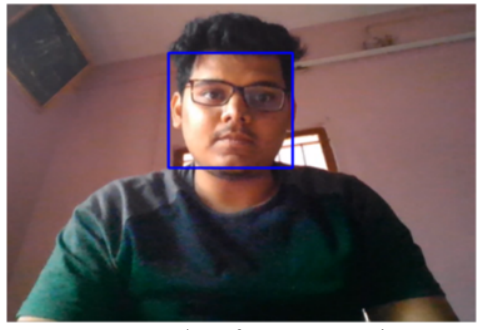

Figure 2. Results of Face Detection

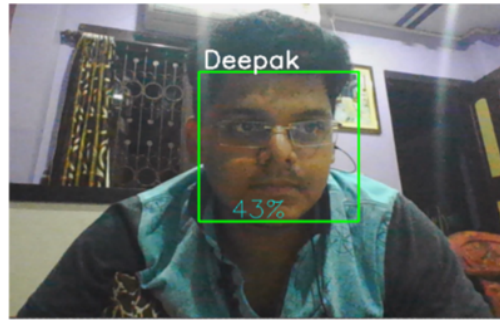

Figure 3. Results of Face Recognition

\subsection{Face Recognition}

In order for system to start recognizing faces, cv2 and numpy libraries are imported. cv2 library contains a Local Binary Pattern Histogram (LBPH) Face Recognizer class. In this class, create method is used to create a recognizer for the model, the recognizer will read the trainer.yml file and based on the training file the output is predicted using the predict function of the recogniser. From the current model we got an accuracy of $43 \%$. Figure 3 shows the results of face recognition. For testing of our model we are using live images.

\subsection{Intranet Connectivity}

In Android Studio, firstly WifiManger, Formatter and AppCompatActivity packages are imported, In order to get the IP Adress of connected WIFI we first have to invoke the WiFi Manager using getSystemService() method. After invoking, connectionInfo method is used to get all the information regarding the current connected WIFI. IP Address is extracted from the received information and it is then formatted using formatIpAddress() method. The resulted IP Address is then checked against the predefined IP Address and if it's a match the intent of the system moves to Login activity otherwise an error is displayed.

For marking daily attendance the user opens the application, during the splash screen the requirement for proper connectivity is checked if the user is in proper network area then the intent goes to main activity where the user captures his/her photo using front camera, then this photo is checked with the existing images in the databases and if any image is matched then the name of the person is shown in the screen. Now the user has to click on the submit button. One the button is clicked the intent of the application goes to success page where a message is shown to the user. Now the user's attendance is marked and he/she can close the app. If during splash screen the proper network condition is not matched then the intent of the application goes to error page 
and an appropriate message is shown to the user. So the user has to go in the prescribed area to mark the daily attendance.

\section{Conclusion}

In the conclusion, we can say that the online attendance marking system that we build can ease the hassle that occurs in the morning and evening and it can also prevent proxy attendance. The most important benefit of this is that it can be used as an alternative to the fingerprint scanner in this COVID-19 pandemic and even after it. But we do have some computational limitations and with higher computational power more accurate and better results can be found. This application currently runs on Android based smartphone but can be extended further on other platform based smartphones. Since we have taken a small dataset of 1500 images in consideration and used HAAR Cascade for face detection and recognition process therefore we got an accuracy of $43 \%$ but with larger data set and advance algorithms like CNN, accuracy of the system can be further increased.

\section{Acknowledgements}

We would like to thank all the family members of one our author Deepak Sharma for their contribution in building the dataset for training of our model. We have used real time dataset in our work.

\section{References}

[1] L. Cuimei, Q. Zhiliang, J. Nan, and W. Jianhua. Human face detection algorithm via haar cascade classi-fier combined with three additional classifiers. In 2017 13th IEEE International Conference on Electronic Measurement Instruments (ICEMI), pages 483-487, 2017.

[2] Y. Fujisawa, Y. Otomo, Y. Ogata, Y. Nakamura, R. Fujita, Y. Ishitsuka, R. Watanabe, N. Okiyama, K. Ohara, and M. Fujimoto. Deep-learning-based, computer-aided classifier developed with a small dataset of clinical images surpasses board-certified dermatologists in skin tumour diagnosis. British Jour-nal of Dermatology, 180(2):373-381, 2019.

[3] Nirmalya Kar, Dr. Mrinal Deb Barma, Ashim Saha, and Dwijen Pal. Study of implementing automated attendance system using face recognition technique. International Journal of Computer and Communication Engineering, pages 100-103, 012012.

[4] Visar Shehu and Agni Dika. Using real time computer vision algorithms in automatic attendance management systems. pages $397-402,072010$.

[5] S. Thakur, J. K. Sing, D. K. Basu, M. Nasipuri, and M. Kundu. Face recognition using principal component analysis and rbf neural networks. In 2008 First International Conference on Emerging Trends in Engineering and Technology, pages 695-700, 2008.

[6] E. Varadharajan, R. Dharani, S. Jeevitha, B. Kavinmathi, and S. Hemalatha. Automatic attendance man-agement system using face detection. In 2016 Online International Conference on Green Engineering and Technologies (IC-GET), pages 1-3, 2016.

[7] P. Wagh, R. Thakare, J. Chaudhari, and S. Patil. Attendance system based on face recognition using eigen face and pca algorithms. In 2015 International Conference on Green Computing and Internet of Things (ICGCIoT), pages 303-308, 2015. 